\title{
E2F transcription factor 8 promotes cell proliferation via CCNDI/p2I in esophageal squamous cell carcinoma
}

This article was published in the following Dove Press journal:

OncoTargets and Therapy

\section{Huiwen Chang \\ Jianxiang Song \\ jixiang $\mathrm{Wu}$ \\ Yajun Zhang}

Department of Cardiothoracic Surgery, Yancheng Third People's Hospital, The Affiliated Yancheng Hospital of Southeast University, Yancheng, Jiangsu 22400 I, PR China
Correspondence: Yajun Zhang Department of Cardiothoracic Surgery, Yancheng Third People's Hospital, The Affiliated Yancheng Hospital of Southeast University, No 75 Juchang Road, Yancheng 22400 I Jiangsu, PR China Tel +86 I37 70067799 Email ycdsrmyyzyj@।63.com
Purpose: E2F transcription factor $8(E 2 F 8)$ is a novel member of the E2F family, but its function in esophageal squamous cell carcinoma (ESCC) remains unclear. This study aimed to research the function of $E 2 F 8$ in ESCC.

Materials and methods: We used quantitative real-time PCR and Western blot analyses to detect the expression pattern of E2F8 in ESCC. The effects of E2F8 on proliferation were investigated by Cell Counting Kit- 8 , 5-ethynyl-2'-deoxyuridine, and colony formation assays. We also confirmed the function of $E 2 F 8$ in vivo.

Results: E2F8 expression was upregulated in ESCC, and promoted cell proliferation and influenced the expression of CCND1/p21. Downregulation of E2F8 expression inhibited cell proliferation in vivo.

Conclusion: $E 2 F 8$ was identified as a new potential oncogene in ESCC.

Keywords: $E 2 F 8$, cell cycle, ESCC, cell proliferation

\section{Introduction}

Esophageal cancer is a common kind of malignant tumor with a high incidence and mortality. Most patients are diagnosed at an advanced stage, but even at the early stage of diagnosis, the prognosis is still dismal. Almost $90 \%$ of esophageal cancers in Asia are esophageal squamous cell carcinoma (ESCC). ${ }^{1}$ Great developments have recently been made in treatments for ESCC, and surgical techniques and perioperative treatments have advanced; however, the prognosis of ESCC remains poor. ${ }^{2}$ The main reasons for the late diagnosis and poor prognosis may be due to the mechanism of ESCC still remaining unknown and the lack of efficient biomarkers for diagnosis and therapy. Therefore, it is urgent to find the key markers and basic mechanisms that can affect the response of ESCC to chemotherapy.

E2F family members can function as transcription factors that bind to target promoters to affect their expression. ${ }^{3}$ As far as we know, eight members have been identified as in this family (E2F1-8). E2F1-3 were proven to be transcriptional activators, while E2F4-7 were shown to inhibit the transcriptional expression of downstream target genes. ${ }^{4-6}$ However, the functions of $E 2 F 8$, a novel member, still remain unclear. It was reported that the combination of $E 2 F 8$ and E2F7 is essential for embryonic development in mice, ${ }^{7,8}$ angiogenesis, ${ }^{9}$ and lymphangiogenesis ${ }^{10}$ in zebrafish. Moreover, previous studies demonstrated that E2F8 exhibited crucial functions in different kinds of cancers. Sun et al discovered that $E 2 F 8$ influenced the cell cycle and further promoted papillary thyroid cancer development. ${ }^{11}$ Deng et al 
found that $E 2 F 8$ may work as a potential therapeutic target in hepatocellular carcinoma. ${ }^{12}$ All these studies indicated that $E 2 F 8$ could act as a potential oncogene in several cancers. However, the features of E2F8 in ESCC have not been investigated until now. In the present study, we identified that $\mathrm{E} 2 \mathrm{~F} 8$ was upregulated in ESCC tissue and promoted the proliferation of ESCC cells by regulating CCND1/p21.

In conclusion, our findings demonstrated that $E 2 F 8$ exhibited important functions affecting ESCC proliferation and regulating the cell cycle, indicating that $E 2 F 8$ could be a potential novel therapeutic target in ESCC.

\section{Materials and methods}

Tissue specimens of 60 paired tumors and their adjacent normal tissue were obtained from patients who were diagnosed with ESCC and underwent resection surgery between 2014 and 2015 at the Department of Cardiothoracic Surgery, Yancheng Third People's Hospital, The Affiliated Yancheng Hospital of Southeast University, Jiangsu, PR China. The pathological diagnosis was made by the Department of Pathology in Yancheng Third People's Hospital, Jiangsu, PR China. Informed consent was obtained from the patients. If the patients were not able to answer or sign, consent was obtained from their relatives before the collection of specimens. All the consents were documented with written informed consent forms and were conducted in accordance with the Declaration of Helsinki. The study was approved by the Medical Ethical Committee of the Medical School of Southeast University. Samples were stored at $-70^{\circ} \mathrm{C}$ after resection until RNA extraction.

\section{ESCC cell lines and cell culture}

ESCC cell lines, including TE-1, KYSE-150, and TE-10, were purchased from American Type Culture Collection (ATCC, Manassas, MA, USA). Normal human esophageal epithelial cells (HEEC) and ESCC cells, including the EC-1 and EC-109 cell lines, were obtained from the Cell Bank of the Chinese Academy of Sciences (Shanghai, PR China). The culture conditions were as follows: Dulbecco's Modified Eagle's Medium (Wisent Bioproducts, St-Bruno, QC, Canada) supplemented with 10\% FBS (Wisent Bioproducts), $100 \mathrm{U} / \mathrm{mL}$ penicillin, and $100 \mu \mathrm{g} / \mathrm{mL}$ streptomycin in a moist incubator (stabilized at $5 \% \mathrm{CO}_{2}$ and $37^{\circ} \mathrm{C}$ ).

\section{Knockdown and overexpression of E2F8}

To knockdown $E 2 F 8$, siRNAs targeting the coding region of E2F8 were obtained from GenePharma (Shanghai, PR China), and siRNA transfection reagent (Thermo Fisher
Scientific, Waltham, MA, USA) was utilized according to the manufacturer's protocols. An E2F8 inhibitor lentivirus $(s h E 2 F 8)$ was designed based on the sequence of siRNA for $E 2 F 8$. Nontargeting control siRNA was used as a negative control. The sequences used were as follows: siRNA for $E 2 F 8$ : 5'-GGCCAAAGACUGUAUACACTT-3' (sense), 5'-GUGUAUACAGUCUUUGGCCTT-3' (antisense); and nontargeting control siRNA (NC): $5^{\prime}$-UUCUC CGAACGUGUCACGUTT-3' (sense), 5'-ACGUGAC ACGUUCGGAGAATT-3' (antisense). To overexpress $E 2 F 8$, ESCC cell lines were transfected with the $E 2 F 8$-overexpressing recombinant vector pcDNA3.1-E2F8 (GenePharma); the empty plasmid was utilized as the negative control. The primer to overexpress $E 2 F 8$ was designed as follows: 5'-CGGGATCCGAGGAATTTACAGAATGG AGAAC-3' (forward); 5'-CCCGCTCTAGATTAATGGAC ATCCTCTGTTGAGACTTC-3' (reverse).

\section{Quantitative real-time PCR (qRT-PCR)}

Following the manufacturer's instructions, total RNA was extracted from ESCC tissue and cells with TRIzol reagent (Thermo Fisher Scientific). For qRT-PCR of the mRNA content, total RNA was reverse transcribed into cDNA with a PrimeScript RT reagent kit (Takara, Shiga, Japan). The reactions were performed using a 7,500 Real-time PCR System (Thermo Fisher Scientific) with a SYBR Premix Ex Taq kit (Takara). The specific primers for the target mRNA and internal control were designed as follows: $E 2 F 8$ forward: 5'-CCAACCCTGCTGTGAATA-3' and $E 2 F 8$ reverse: $5^{\prime}$-TTTCTGGCTCATTACCCT-3'; and $\beta$-actin forward: $5^{\prime}$-GCATCGTCACCAACTGGGAC-3' and $\beta$-actin reverse: $5^{\prime}$-ACCTGGCCGTCAGGCAGCTC- $3^{\prime}$. The expression pattern was normalized to the internal control ( $\beta$-actin), and the results are shown in the form of relative expression and were calculated by the $2^{-\Delta \Delta \mathrm{CT}}$ method.

\section{Colony formation assay}

Under the culture conditions described earlier, 500 cells/well were seeded into six-well plates. After 1 week, each well underwent the following procedure: washed with PBS for three times at room temperature, fixed in ethyl alcohol for 30 seconds, and finally stained with crystal violet. Colonies were counted with the naked eye after rinsing with PBS.

\section{Cell proliferation assay}

A Cell Counting Kit-8 assay (CCK-8; Dojindo, Tokyo, Japan) was used to detect cell proliferation according to the manufacturer's instructions. A total of 2,000 cells/well 
were seeded into 96 -well plates and cultured as previously described. Every 24 hours, $10 \mu \mathrm{L}$ of CCK-8 solution was added to the serum-free medium. After 2 hours of incubation, the absorbance was detected by a microplate reader at a test wavelength of $450 \mathrm{~nm}$ and a reference wavelength of $630 \mathrm{~nm}$.

\section{5-Ethynyl-2'-deoxyuridine (EdU) assay} An EdU assay kit (RiboBio, Guangzhou, PR China) was used to measure cell proliferation. The cells were inoculated into 24 -well plates $\left(2 \times 10^{4}\right.$ cells/hole $)$ and cultured for 24 hours with DMEM (10\% FBS) before adding EdU $(50 \mu \mathrm{M})$. According to the protocols, cells were incubated at $37^{\circ} \mathrm{C}$ for 2 hours, fixed in $4 \%$ formaldehyde for 30 minutes, and permeabilized at room temperature for 10 minutes with $0.5 \%$ Triton X-100. After washing with PBS, $1 \times$ ApolloR reaction cocktail $(400 \mu \mathrm{L})$ was added to the EdU mixture for 30 minutes. Subsequently, Hoechest33342 (400 $\mu \mathrm{L})$ was added for 30 minutes to visualize the nuclei. Cell images were obtained under a Nikon microscope (Nikon Corporation, Tokyo, Japan). Proliferation was analyzed using the average number of cells in three fields of vision for each sample.

\section{Cell cycle analysis}

First, the transfected cells were digested with trypsin and then centrifuged for 5 minutes at 1,200 rpm. After washing with PBS twice, the cells were immobilized overnight in $75 \%$ ethanol at $-20^{\circ} \mathrm{C}$. The cells were washed with PBS twice before detection, fixed with $500 \mu \mathrm{L}$ of propidium iodide staining solution, and incubated at room temperature for 15 minutes. A BD FACSCanto II (BD Biosciences, San Jose, CA, USA) flow cytometer and FlowJo software were used to analyze the cell cycle.

\section{Western blot analysis}

According to the manufacturer's instructions, a RIPA kit (Beyotime, Shanghai, PR China) was used to extract proteins from ESCC cells and tissue. Based on the molecular weight of each protein on the twelve alkyl SDS-PAGE and utilizing method of Electrophoresis (EMD Millipore, Bedford, MA, USA). We used 5\% nonfat powdered milk in Tris-buffered saline to block the membrane for 2 hours. Then, the membranes were incubated overnight with a specific antibody at $4^{\circ} \mathrm{C}$. After rinsing in TBST (Tris-buffered saline containing $0.05 \%$ Tween-20) for three times (10 minutes each time), membranes were incubated in secondary antibody solutions (rabbit or mouse) at room temperature for 2 hours and then washed again (three times, 10 minutes each time). Protein expression levels were detected by ECL Plus (EMD Millipore) with a Bio-Imaging System. The following primary antibodies were used: anti-E2F8, anti-CCND1, and anti-p21; anti-GAPDH was used as an internal control.

\section{Tumor xenografts in a nude mouse model}

This study was conducted in accordance with the institutional standard guidelines of Southeast University for animal experiments and was approved by the Animal Ethics Committee of Southeast University. Male BALB/c nude mice (aged 4 weeks) were purchased from the Yancheng Third People's Hospital, The Affiliated Yancheng Hospital of Southeast University, Jiangsu, PR China. A total of $2 \times 10^{6}$ transfected cells were injected bilaterally and subcutaneously into the flanks of the nude mice. Every 5 days, the tumors were measured with Vernier calipers, and euthanasia was carried out after 3 weeks. The formula used to calculate the tumor volumes was as follows: volume $=\left(\right.$ width $^{2} \times$ length $) / 2$.

\section{Statistical analysis}

All experiments in this research were performed in triplicate independently. All analyses were performed using SPSS 19.0 software. The data are shown as the mean \pm SD. Student's unpaired $t$-test was used to determine any significant differences in the results. The following indicators were used for data that were considered statistically significant: * indicates $P<0.05$, ** indicates $P<0.01$, and *** indicates $P<0.001$.

\section{Results \\ E2F8 was upregulated in ESCC tissue and ESCC cell lines}

To identify the expression pattern of E2F8 in ESCC, 60 paired ESCC tissue and adjacent normal tissue samples were obtained. Two methods, qRT-PCR and Western blotting, were utilized to detect the expression of E2F8. The qRTPCR results showed that $E 2 F 8$ expression was upregulated in ESCC tumor tissue compared to adjacent normal tissue in the 60 paired ESCC tumor tissue and adjacent normal tissue samples. E2F8 protein expression was also upregulated in six paired ESCC tumor tissue and adjacent normal tissue samples assessed by Western blotting (Figure 1A). The expression levels of E2F8 in ESCC cell lines, including EC-1, TE-1, EC-109, KYSE-150, and TE-10, were further investigated. Compared to normal esophageal epithelial HEEC cells, ESCC cells lines showed upregulated E2F8 expression by both qRT-PCR and Western blotting (Figure 1B). In summary, E2F8 expression was upregulated in ESCC. 

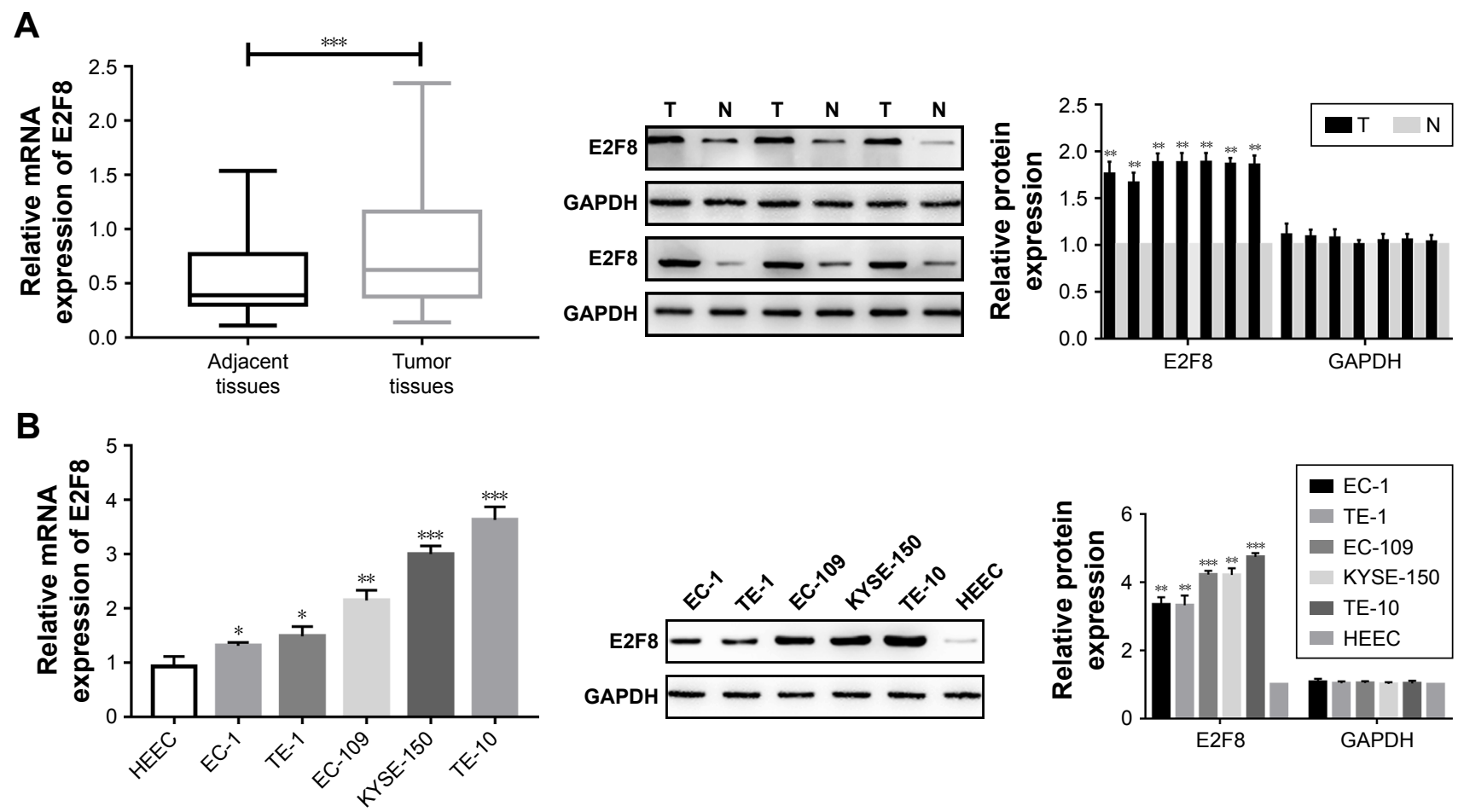

Figure I E2F8 is upregulated in ESCC.

Notes: (A) Expression pattern of E2F8 in 60 paired ESCC tissues and adjacent normal tissues was detected by qRT-PCR/Western blot. (B) Expression pattern of E2F8 in ESCC cell lines and normal human esophageal epithelial cells (HEEC) was detected by qRT-PCR and Western blot $(* P<0.05$, $* * P<0.0 \mathrm{I}, * * * P<0.00 \mathrm{I})$.

Abbreviations: ESCC, esophageal squamous cell carcinoma; $\mathrm{T}$, tumor tissues; $\mathrm{N}$, adjacent non-tumor tissues; qRT-PCR, quantitative real-time PCR.

\section{E2F8 promotes ESCC cell proliferation and arrests the cell cycle in $\mathrm{S}$ phase}

Two cell lines were selected based on their E2F8 expression patterns. E2F8 was overexpressed in EC-1 cells, which had the lowest expression level among the five ESCC cell lines studied, and $E 2 F 8$ was downregulated in TE-10 cells, which had the highest expression level among the five ESCC cell lines studied. Using qRT-PCR and Western blotting, the transfection efficiencies were confirmed. The results indicated that $E 2 F 8$ expression was successfully upregulated in EC-1 cells and downregulated in TE-10 cells (Figure 2A). To investigate the effects of E2F8 on proliferation, three assays, CCK-8, colony formation, and EdU assays, were conducted. CCK- 8 assays showed that downregulating $E 2 F 8$ expression decreased the proliferation ability of TE-10 cells, while upregulating E2F8 expression increased the proliferation ability of EC-1 cells (Figure 2B). Images of representative plates with colonies (Figure 2C) similarly showed that the downregulation of $E 2 F 8$ expression in TE-10 cells decreased their colony formation ability compared to that of the control group. Upregulated $E 2 F 8$ expression in EC-1 cells increased their colony formation ability compared to that of the NC group (Figure 2C). The results of EdU assays showed that the rate at which TE-10 cells incorporated EdU in the downregulated $E 2 F 8$ group distinctly decreased compared to the rate in the control group; however, the opposite results were detected in the EC-1 cell lines when comparing the rate at which cells incorporated EdU in the upregulated $E 2 F 8$ group to that of the NC group (Figure 2D). Cells incorporating EdU were considered to be proliferating cells. Flow cytometry was performed to analyze the effects of dysregulated $E 2 F 8$ expression on the cell cycle. The results showed that downregulating $E 2 F 8$ expression in TE-10 cells produced a significant increase in the percentage of cells in the G0/G1 phase, while upregulating E2F8 in EC-1 cells resulted in cell cycle arrest in $\mathrm{S}$ phase (Figure 2E). These findings demonstrated that $E 2 F 8$ promoted ESCC cell proliferation and arrested the cell cycle at $\mathrm{S}$ phase.

\section{E2F8 influences CCNDI/p2 I}

Previous studies have revealed that E2F8 influences the cell cycle by interacting with key factors. ${ }^{11}$ We wondered whether E2F8 could affect these factors in ESCC. qRT-PCR and Western blotting were performed to detect the expression patterns of CCND1 and p21 in the two pairs of genetically engineered cell lines. The results demonstrated that downregulating E2F8 expression decreased the expression 
A

TE-10
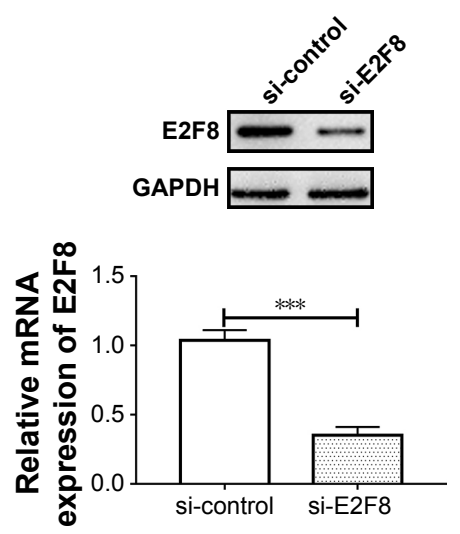

B

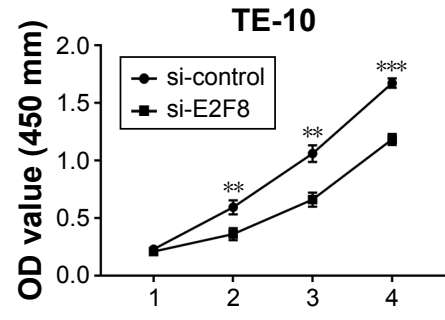

C

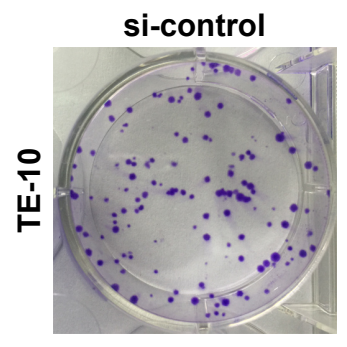

si-E2F8

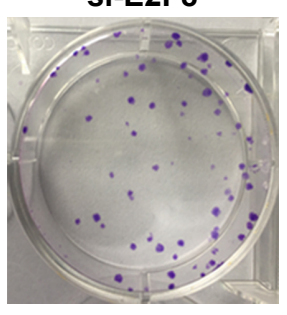

NC
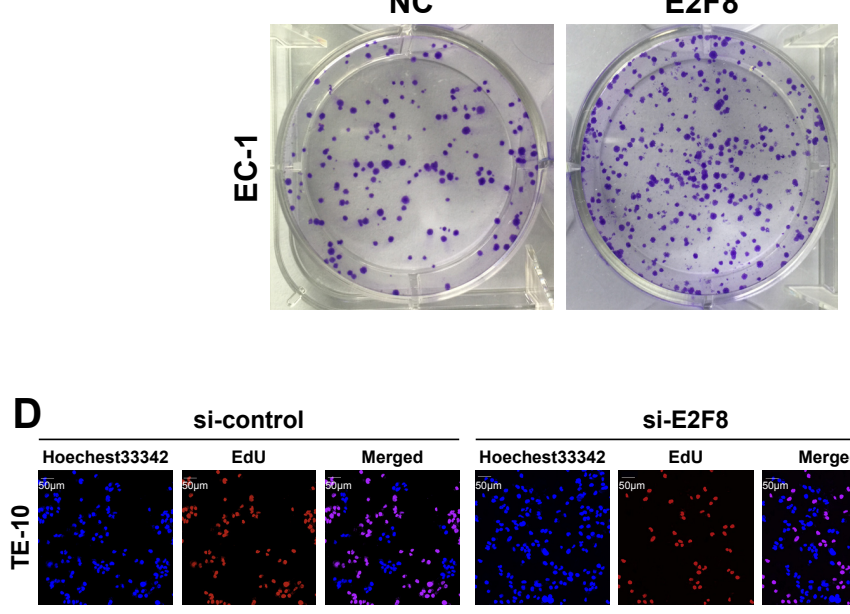
si-control

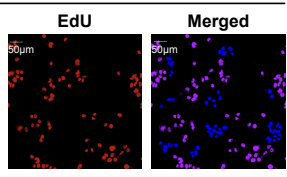

NC
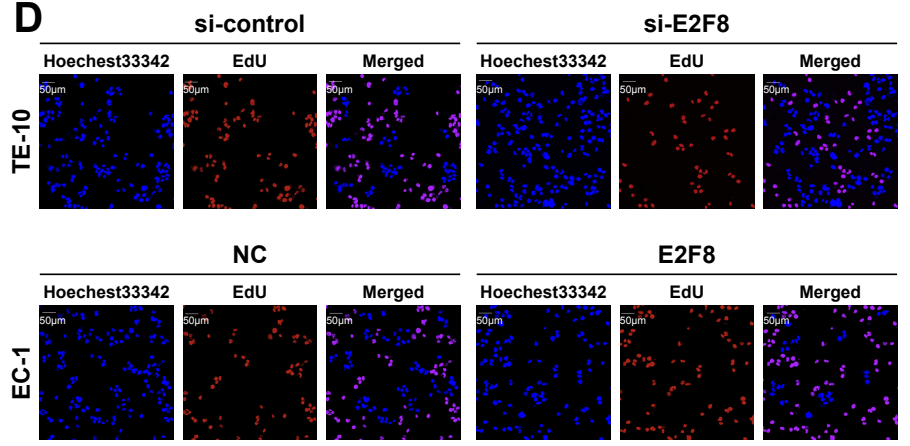

E2F8

EC-1

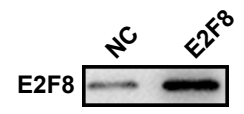

GAPDH
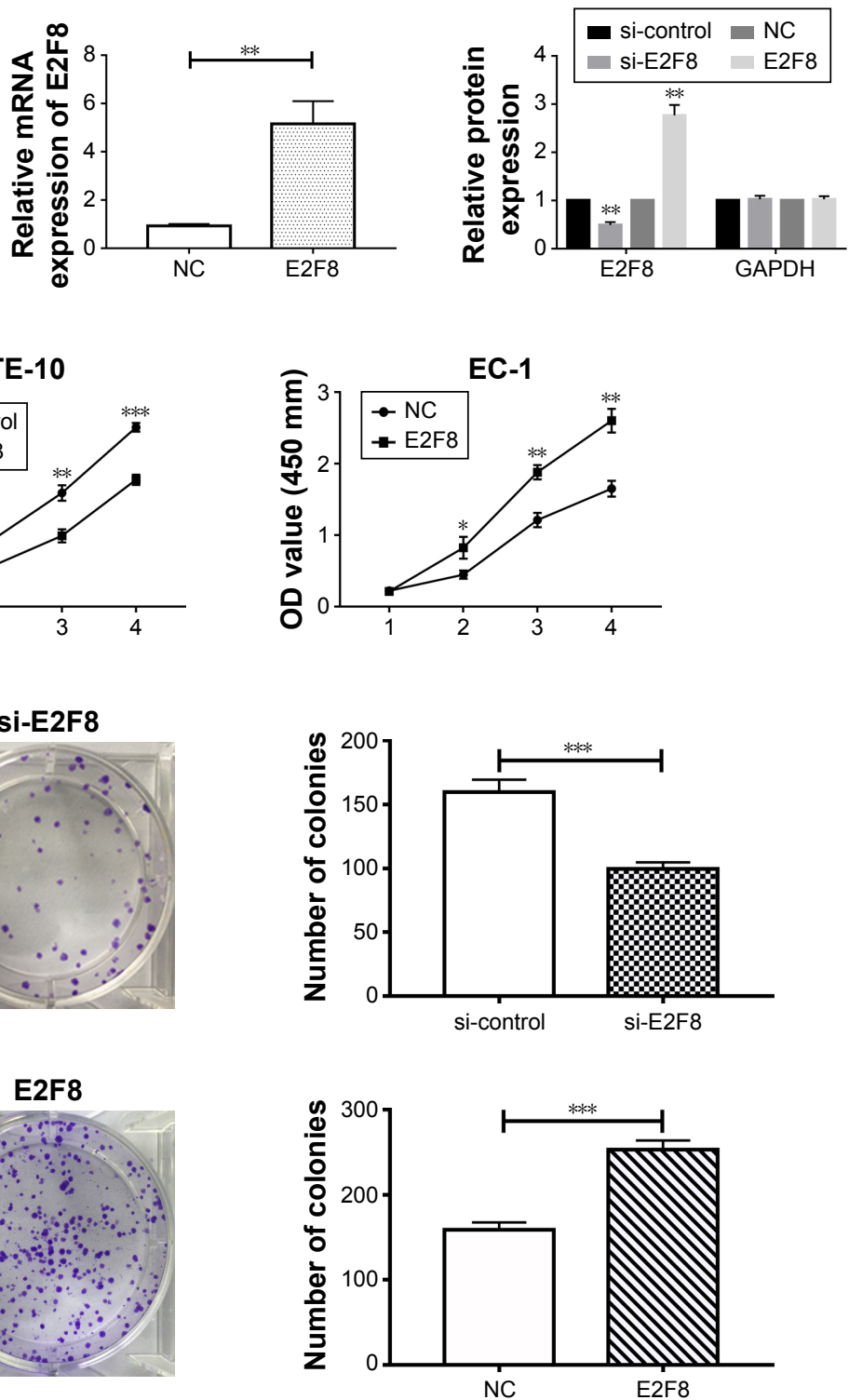

Figure 2 (Continued)
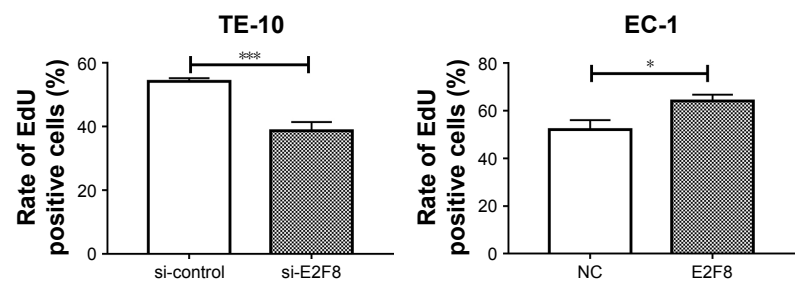
E
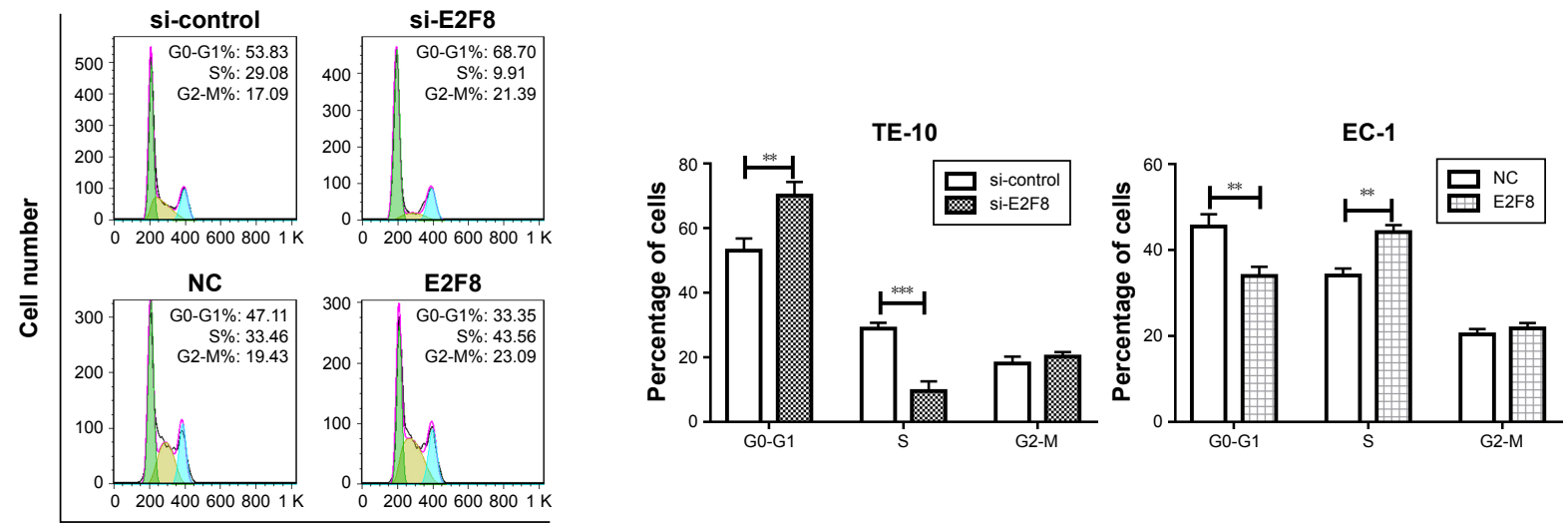

DNA contents

Figure 2 E2F8 promotes ESCC cell proliferation and arrests cell cycle at $\mathrm{S}$ phase.

Notes: (A) qRT-PCR was used to confirm the transfection efficiency of si-E2F8 in TE-10 and that of pcDNA3.I-E2F8 in EC-I. (B-D) Proliferation effects of downregulated E2F8 in TE- 10 and those of upregulated E2F8 in EC-I compared to control group were detected by CCK-8, colony formation, and EdU assays. (E) Effects of downregulated E2F8 on regulating cell cycle in TE-I0 and effects of upregulated E2F8 in EC-I compared to control group were detected by flow cytometry $(* P<0.05$, $* * P<0.0 \mathrm{I}$, $* * * P<0.00 \mathrm{I})$. Abbreviations: ESCC, esophageal squamous cell carcinoma; qRT-PCR, quantitative real-time PCR; CCK-8, Cell Counting Kit-8; EdU, 5-ethynyl-2'-deoxyuridine; NC, negative control.

of CCND1 and increased the expression of p21 in TE-10 cells, while upregulating E2F8 expression increased the expression of CCND1 and decreased the expression of p21 in EC-1 cells (Figure 3).

\section{E2F8 promotes tumorigenicity in vivo}

To reveal the effects of $E 2 F 8$ on tumorigenicity in vivo, TE-10 cells treated as previously described were injected into the flanks of nude mice to generate tumors ectopically.
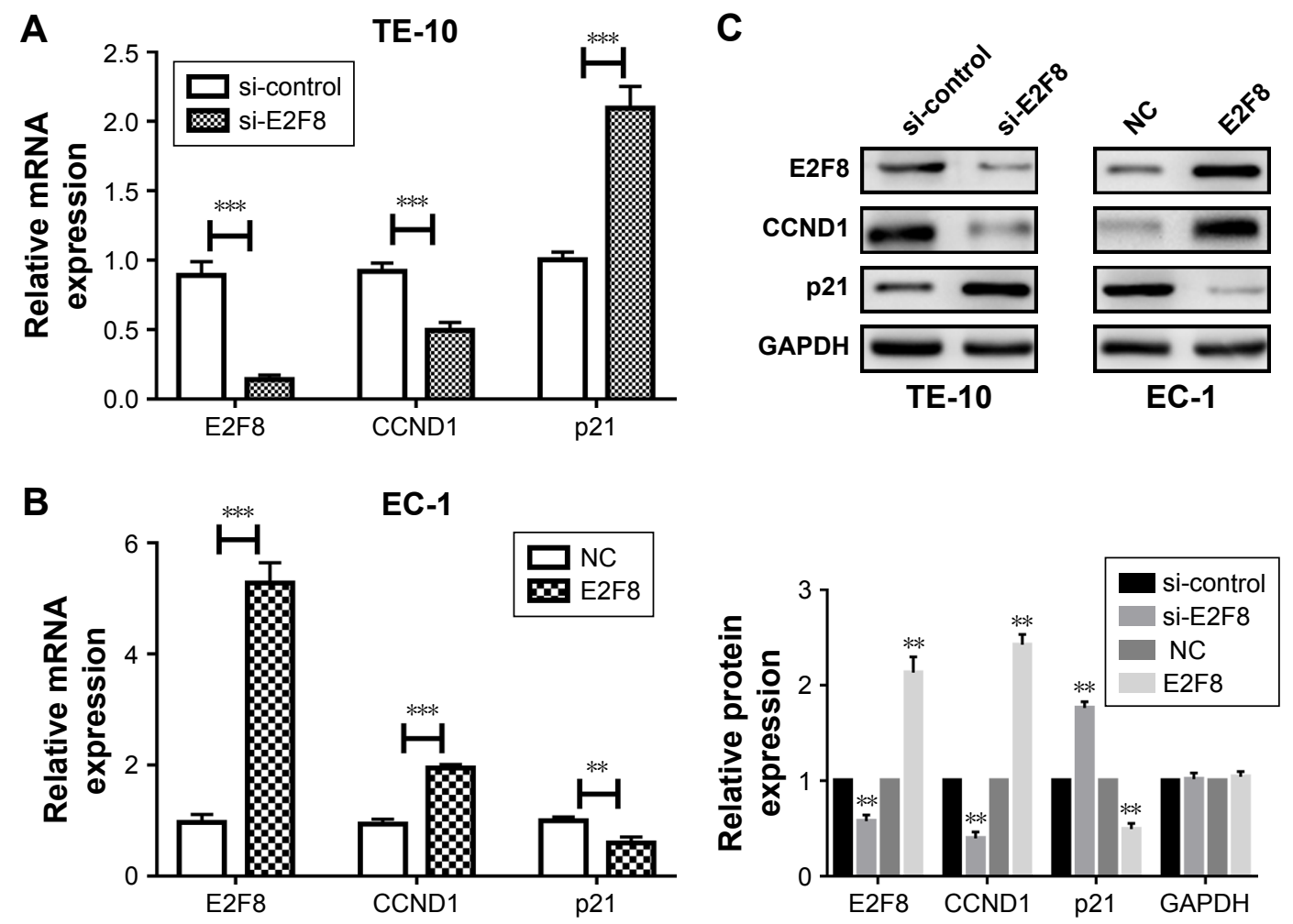

Figure 3 Effects of E2F8 on the expression of CCNDI/p21.

Notes: (A, B) Effects of downregulated E2F8 on regulating the expression of CCNDI/p2I in TE- 10 cells and effects of upregulated E2F8 in EC-I cells compared to control group were detected by qRT-PCR. (C) Effects of downregulated E2F8 on regulating the expression of CCNDI/P2I in TE-I0 and effects of upregulated E2F8 in EC-I compared to control group were detected by Western blot $(* * P<0.01$, *** $P<0.00$ I).

Abbreviations: qRT-PCR, quantitative real-time PCR; NC, negative control. 

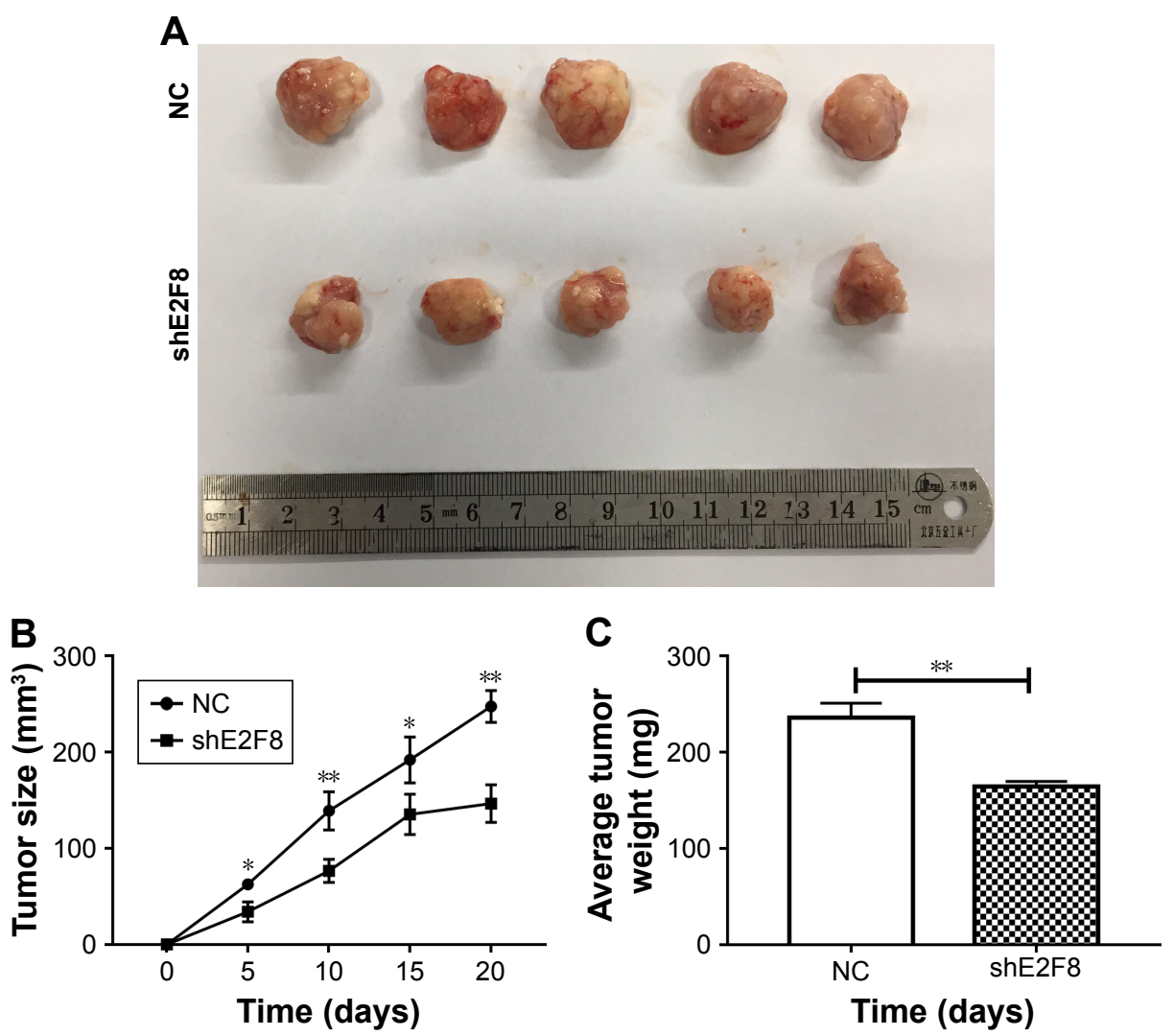

Figure 4 Downregulated E2F8 inhibits tumorigenicity in vivo.

Notes: (A) Tumors generated by TE-10 which were transfected with shE2F8 were compared to tumors in the control group. (B) TE- 10 transfected with shE2F8 generated smaller tumors. (C) TE-I0 transfected with shE2F8 generated lighter tumors $(* P<0.05, * * P<0.01)$.

Abbreviation: NC, negative control.

The results showed that compared to the TE-10 control cells, the cells transfected with $s h E 2 F 8$ generated smaller and lighter tumors (Figure 4).

\section{Discussion}

Esophageal cancer is a malignant tumor with high morbidity and mortality due to recurrence, metastasis, and drug resistance. The main cause of these phenomena is the lack of knowledge about the disease mechanism; thus, it is imperative to reveal the potential mechanism of this disease. It may be much easier to diagnose and treat this kind of disease with identification of effective biomarkers or oncogenes. Among the various kinds of esophageal cancer, ESCC is the most common. ${ }^{1}$ Thus, in the present study, we focused on researching the mechanism and potential biomarkers of ESCC.

mRNA is a large class of RNA molecules that transfer genetic information from DNA to ribosomes; they specify the amino acid sequences of the protein products based on the expression of genes. ${ }^{13}$ Regarding cancer treatment, mRNAs have been widely investigated as biomarkers in multiple cancers. Zhang et al revealed that TRIM27 functions as an oncogene by activating epithelial-mesenchymal transition and p-AKT in colorectal cancer. ${ }^{14}$ Sun et al proved that TRIM59 facilitated the proliferation of colorectal cancer and promoted metastasis via the $\mathrm{PI} 3 \mathrm{~K} / \mathrm{AKT}$ pathway. ${ }^{15}$

The E2F transcription factor family is of paramount importance for coordinating cell cycle progression. Among the known E2F family members, eight members, E2F1-8, have been recognized. In general, E2F1-3 are considered as transcriptional activators, whereas E2F4-7 play inhibitory roles in the transcriptional expression of downstream target genes. ${ }^{4-6}$ All these observations demonstrated that E2F transcription factors have significant effects on cell fate. Thus, it is urgent to identify the specific role for each E2F family member in specific malignancies. E2F1-7 were described in previous studies. ${ }^{4-6}$ In the present study, we focused on $E 2 F 8$, a new member of the E2F family. E2F8 has been previously researched and shown to regulate the cell cycle in several cancers. Zhang et al discovered that E2F8 expression is upregulated in colorectal cancer. ${ }^{16}$ Jin et al discovered that by suppressing $E 2 F 8$, metformin can induce cell cycle arrest in the G1 phase in lung cancer cells. ${ }^{17}$ Sun et al found that $E 2 F 8$ can promote papillary thyroid cancer progression by regulating the cell cycle. ${ }^{11}$ Ye et al indicated that upregulating E2F8 
expression modulates the G1/S phase transition and further promotes cell proliferation in breast cancer. ${ }^{18}$ However, the function of $E 2 F 8$ still remains unknown in ESCC. In the present study, the expression pattern of E2F8 in ESCC was detected by qRT-PCR and Western blotting. The results showed that E2F8 expression was upregulated in ESCC tissue and cell lines. Transfected cell lines that upregulate or downregulate E2F8 expression were constructed. Using these cell lines, the effects of $E 2 F 8$ on cell proliferation were investigated by CCK-8, colony formation, and EdU assays. Persistent proliferation is considered a hallmark of cancer. ${ }^{19}$ Our studies demonstrated that E2F8 expression was upregulated in ESCC, and upregulated $E 2 F 8$ expression promoted the proliferation of ESCC cells. In contrast, downregulated E2F8 expression presented the opposite effects by inhibiting ESCC cell proliferation. These findings indicated that $E 2 F 8$ was dysregulated in ESCC and may play a significant role in ESCC by influencing cell proliferation. Cell proliferation is the main aspect that is controlled by the cell cycle. In general, the cell cycle is dysregulated in cancer cells, which destroys cell density homeostasis and leads to uncontrolled cell proliferation. ${ }^{20} \mathrm{We}$ wondered whether $E 2 F 8$ could affect the cell cycle. Using flow cytometry, upregulated $E 2 F 8$ expression was discovered to arrest the cell cycle in $\mathrm{S}$ phase. Previous studies proved that the E2F8 gene, located on chromosome $11 \mathrm{p} 15$, encodes a family member of the transcription factors that regulate the genes required for progression through the cell cycle, and $E 2 F 8$ could regulate progression from $\mathrm{G} 1$ to $\mathrm{S}$ phase by ensuring that the nucleus divides at the proper time. ${ }^{21}$ Our results were consistent with previous findings, further indicating that $E 2 F 8$ is an indispensable cell cycle regulatory factor in ESCC. We further detected the relationship between E2F8 and CCND1/ p21, which were identified as key cell cycle factors. Through Western blotting, E2F8 was revealed to have the ability to influence $\mathrm{p} 21$, which can be a universal inhibitor ${ }^{22}$ of cyclin kinases and CCND1, which was identified as a key factor in regulating the cell cycle. ${ }^{23}$ It can be further deduced that $E 2 F 8$ might be a transcriptional repressor of $\mathrm{p} 21$ and transcriptional activator of CCND1 in ESCC. CCND1 functions as an oncogene that is involved in many cancers by regulating the $\mathrm{G} 1$ to $\mathrm{S}$ phase transition of the cell cycle, while p21 acts as a master effector in multiple tumor suppressor pathways. Thus, $E 2 F 8$ might play critical roles in regulating the cell cycle in ESCC by influencing CCND1/p21. However, the detailed mechanism of $E 2 F 8$-mediated regulation of the cell cycle still needs further investigation. Taken together, through the activities of $E 2 F 8$, CCND1 and p21 could be activated or suppressed and the proliferative abilities of cells could be changed. Finally, E2F8 expression promoted ESCC cell proliferation in vivo.
In conclusion, our study identified $E 2 F 8$ as an oncogene that promoted cell proliferation by CCND1/p21 in vitro and in vivo.

\section{Author contributions}

All authors contributed to data analysis, drafting or revising the article, gave final approval of the version to be published, and agree to be accountable for all aspects of the work.

\section{Disclosure}

The authors report no conflicts of interest in this work.

\section{References}

1. Torre LA, Bray F, Siegel RL, Ferlay J, Lortet-Tieulent J, Jemal A. Global cancer statistics, 2012. CA Cancer J Clin. 2015;65(2):87-108.

2. Koshy M, Esiashvilli N, Landry JC, Thomas CR, Matthews RH. Multiple management modalities in esophageal cancer: combined modality management approaches. Oncologist. 2004;9(2):147-159.

3. Attwooll C, Lazzerini Denchi E, Helin K. The E2F family: specific functions and overlapping interests. EMBO J. 2004;23(24):4709-4716.

4. Degregori J, Johnson DG. Distinct and overlapping roles for E2F family members in transcription, proliferation and apoptosis. Curr Mol Med. 2006;6(7):739-748.

5. Dimova DK, Dyson NJ. The E2F transcriptional network: old acquaintances with new faces. Oncogene. 2005;24(17):2810-2826.

6. Tsantoulis PK, Gorgoulis VG. Involvement of E2F transcription factor family in cancer. Eur J Cancer. 2005;41(16):2403-2414.

7. $\mathrm{LiJ}, \mathrm{Ran} \mathrm{C}, \mathrm{Li}$ E, et al. Synergistic function of E2F7 and E2F8 is essential for cell survival and embryonic development. Dev Cell. 2008;14(1):62-75.

8. Ouseph MM, Li J, Chen HZ, et al. Atypical E2F repressors and activators coordinate placental development. Dev Cell. 2012;22(4):849-862.

9. Weijts BG, Bakker WJ, Cornelissen PW, et al. E2F7 and E2F8 promote angiogenesis through transcriptional activation of VEGFA in cooperation with HIF1. EMBO J. 2012;31(19):3871-3884.

10. Weijts BG, van Impel A, Schulte-Merker S, de Bruin A. Atypical E2fs control lymphangiogenesis through transcriptional regulation of Ccbe1 and Flt4. PLoS One. 2013;8(9):e73693.

11. Sun J, Shi R, Zhao S, et al. E2F8, a direct target of miR-144, promotes papillary thyroid cancer progression via regulating cell cycle. $J$ Exp Clin Cancer Res. 2017;36(1):40.

12. Deng Q, Wang Q, Zong WY, et al. E2F8 contributes to human hepatocellular carcinoma via regulating cell proliferation. Cancer Res. 2010; 70(2):782-791.

13. Sullenger BA, Nair S. From the RNA world to the clinic. Science. 2016; 352(6292):1417-1420.

14. Zhang Y, Feng Y, Ji D, et al. TRIM27 functions as an oncogene by activating epithelial-mesenchymal transition and p-AKT in colorectal cancer. Int J Oncol. 2018;53(2):620-632.

15. Sun Y, Ji B, Feng Y, et al. TRIM59 facilitates the proliferation of colorectal cancer and promotes metastasis via the PI3K/AKT pathway. Oncol Rep. 2017;38(1):43-52.

16. Zhang Z, Li J, Huang Y, et al. Upregulated miR-1258 regulates cell cycle and inhibits cell proliferation by directly targeting E2F8 in CRC. Cell Prolif. 2018:e12505.

17. Jin DH, Kim Y, Lee BB, et al. Metformin induces cell cycle arrest at the G1 phase through E2F8 suppression in lung cancer cells. Oncotarget. 2017;8(60):101509-101519.

18. Ye L, Guo L, He Z, et al. Upregulation of E2F8 promotes cell proliferation and tumorigenicity in breast cancer by modulating $\mathrm{G} 1 / \mathrm{S}$ phase transition. Oncotarget. 2016;7(17):23757-23771.

19. Hanahan D, Weinberg RA. Hallmarks of cancer: the next generation. Cell. 2011;144(5):646-674.

20. Evan GI, Vousden KH, Proliferation VKH. Proliferation, cell cycle and apoptosis in cancer. Nature. 2001;411(6835):342-348. 
21. Christensen J, Cloos P, Toftegaard U, et al. Characterization of E2F8, a novel E2F-like cell-cycle regulated repressor of E2F-activated transcription. Nucleic Acids Res. 2005;33(17):5458-5470.

22. Xiong Y, Hannon GJ, Zhang H, Casso D, Kobayashi R, Beach D. p21 is a universal inhibitor of cyclin kinases. Nature. 1993;366(6456):701-704.
23. Massagué J. G1 cell-cycle control and cancer. Nature. 2004;432(7015): 298-306.

\section{Publish your work in this journal}

OncoTargets and Therapy is an international, peer-reviewed, open access journal focusing on the pathological basis of all cancers, potential targets for therapy and treatment protocols employed to improve the management of cancer patients. The journal also focuses on the impact of management programs and new therapeutic agents and protocols on

\section{Dovepress}

patient perspectives such as quality of life, adherence and satisfaction. The manuscript management system is completely online and includes a very quick and fair peer-review system, which is all easy to use. Visit http://www.dovepress.com/testimonials.php to read real quotes from published authors.

\footnotetext{
Submit your manuscript here: http://www.dovepress.com/oncotargets-and-therapy-journal
} 